\title{
Diagnostic Value of Immunohistochemical Markers in Four-grade Histological Classification of Hepatocellular Carcinoma
}

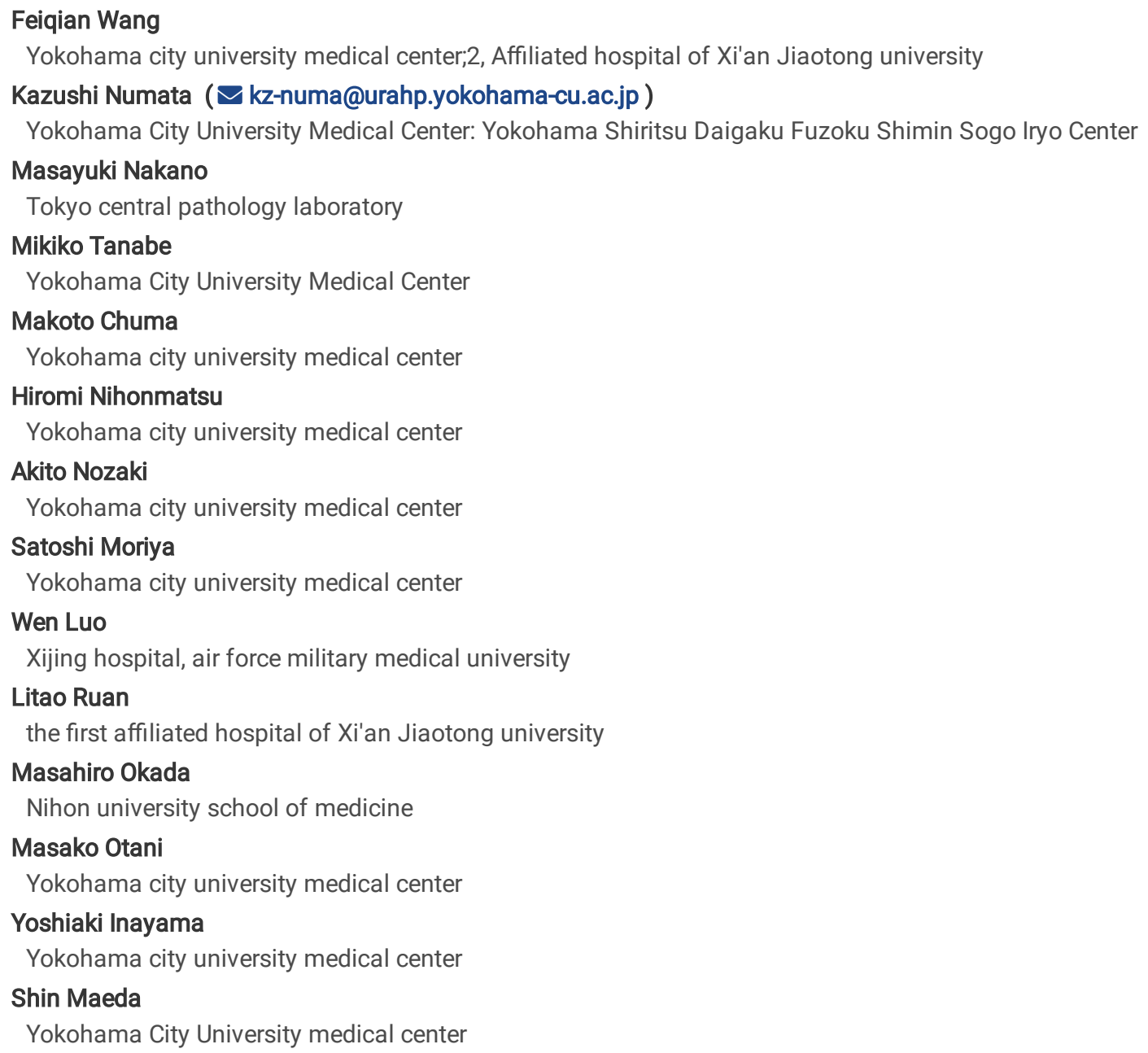

\section{Research}

Keywords: hepatocellular carcinoma, immunohistochemical markers, histological grade, cancer diagnosis

Posted Date: November 2nd, 2020

DOI: https://doi.org/10.21203/rs.3.rs-99711/v1

License: (c) (7) This work is licensed under a Creative Commons Attribution 4.0 International License. Read Full License 


\section{Abstract}

Backgrounds: Accurate differential diagnosis regarding histological grades of hepatocellular carcinoma (HCC) is critical for targeted treatment and prognostic evaluation. However, the currently used descriptive diagnosis of histological grading have to observe tedious item of neovascularization, stromal invasion, cellular and structural atypia, which have drawbacks of subjectivity and error-prone. Immunohistochemical (IHC) markers-based diagnosis only needs to determine whether there is staining in the cell membrane, cytoplasm and / or nucleus. Using IHC markers targeted heat shock protein (HSP)70, glypican (GPC)3, glutamine synthetase (GS), and organic anion transport peptide (OATP), we sought to establish an easy method for the diagnosis of the histopathological grades and further explore the best efficacy by their combined or independent application.

Methods: We retrospectively conducted a study of 157 patients with 200 histologically confirmed HCCs, which were classified into early ( $\mathrm{n}=45$ ), well $(n=31)$, moderately $(n=68)$, and poorly $(n=56)$ differentiated (diff.) HCC. The sensitivity, specificity, accuracy of HSP70, GPC3, GS and OATP on each histological grade were examined.

Results: HSP70 and GPC3 showed difference in most histological grades of HCC $(P<0.05)$. GS distinguished early HCC from three other histological grades $(p<0.01)$. OATP8 only differentiated early HCC from poorly diff. HCC $(P=0.019)$. When any two of the three indicators (HSP, GPC3, and GS) were negatively expressed, the diagnostic efficacy of early HCC was the highest, with an area under the curve (AUC) of 0.802 and accuracy of $80.5 \%$. The optimal efficacy for poorly diff. HCC detection was obtained when both GPC3 and HSP70 were positively expressed (74.4\% accuracy; AUC $=0.703)$. However, for well and moderately diff. HCC, a relative satisfactory AUC value (>0.60) failed to yield either by the independent or combined diagnosis of any $\mathrm{IHC}$ indicators.

Conclusion: Using GS, HSP70, and GPC3, early and poorly diff. HCC can be properly diagnosed. IHC markers might be a potential alternative tool for histological differential diagnosis of HCC

\section{Background}

In the diagnosis of hepatocellular carcinoma (HCC), proper histopathology-based classification is beneficial to make a appropriate therapy plan and predict prognosis [1], mainly because different histological grades of HCC show difference in many aspects of diagnosis, treatment and prognosis. For example, patients with early HCC (eHCC) may have a higher surgical cure rate, a lower recurrence rate, and higher short- and longterm survival rates than those with advanced HCC (adHCC) [2][3][4]. EHCC can be completely cured [5], but poorly differentiated (diff.) HCC exhibits relatively worse recurrence-free and overall survival rates [6]. Compared with patients histologically diagnosed as well diff. HCC, those who diagnosed as moderately diff. HCC tend to have advanced clinical stage of HCC [7].

There is a critical problem of currently used histological grading method for HCC. No matter two- (eHCC and adHCC) [7][8], three- (well, moderately, and poorly) [9][10], four-grade (grade I/II/III/IV) [11][12] classification methods and our newly developed a four-grade histological classification method, which classified HCC as "early/well/moderately/poorly differentiated"[13], consistently adopt diagnostic method to observe the morphological and structural criteria (cellular atypia and structural atypia) of both the lesions and surrounding tissues under the microscope, and thus make a comprehensive diagnosis. In details, hematoxylin-eosin (HE) staining was needed to observe cell density, nuclear / cytoplasmic ratio, cell atypia, nuclear atypia, especially the arrangement of trabeculae; silver staining to observe whether reticular fibers were clear or sparse; CD34 to observe neovascularization; Victoria blue (VB) to observe stromal invasion; and cytokeratin (CK)7 staining to distinguish ductular reaction[14]. Obviously, these observation items are tedious and time-consuming. The identification of trabeculae and neovascularization require a certain pathological knowledge of the readers. In addition, there are more descriptive indicators rather than quantitative indicators in the basis of diagnosis. Therefore, the gold standard of histological grading method is a little subjective and not easy to be popularized in clinical practice. Even worse, identification of stromal invasion of tumoral hepatocytes and tumor boundaries, which are considered as of great importance in histological grading diagnosis of HCC, are sometimes difficult to recognize in specimen obtained by fine-needle aspiration because of its limited amount [15]. All these factors contribute to the challenge and even dilemma for achieving a quick and confirmed histological grading diagnosis.

The novel IHC methods make diagnosis according to whether the tumor cell membrane, nucleus and cytoplasm are stained or not. Readers only need to distinguish the cell membrane, nucleus and cytoplasm, and there is no need to observe the tedious structures such as portal vein area, cytoskeleton, and trabeculae. Therefore, it is easier to observe, faster to obtain results, and require fewer expertise pathological technology of operator, so it is more objective. Most importantly, the reported positive results of heat shock protein 70 (HSP70), glypican 3(GPC3), glutamine synthetase (GS), and organic anion transport peptide 8 (OATP8) [16-18][19], provided a promising insight in feasibility of IHC marker-based HCC diagnosis. Nevertheless, there is very few data available regarding $\mathrm{IHC}$ marker-based histological diagnosis of HCC, not to mention the combined use of IHC markers to achieve best effectiveness for histological diagnosis.

In this regard, taking our four-grade histological classification as a golden standard, we conducted a retrospective research to explore the diagnostic ability of the IHC markers. By introducing an alternative diagnostic criteria by IHC indicator, this study might be beneficial for optimize and popularize the histologcial grading diagnosis of HCC in clinical practise. 


\section{Materials And Methods \\ Patient enrollment}

Between January 2014 and August 2018, we retrospectively enrolled a total of 200 newly discovered and untreated hepatic lesions obtained from 157 consecutive patients. These lesions were definitively diagnosed as HCC and histologically graded after ultrasound-guided biopsy and subsequent IHC staining. Clinical information (gender, age, associated liver disease, Child-Pugh classification), radiological images, and histopathology reports were retrospectively collected from a review of the electronic medical record system, radiology database, and pathology records, respectively, of our hospital. The institutional review board of the Medical Center of Yokohama City University approved this study (approval no. B180200054), and written, informed consent was obtained from all patients. Patients with the following characteristics were excluded from this study: (1)Child Pugh score system classified as grade C; (2)Insufficient specimen from the target lesion, making it impossible to obtain a definitive pathological diagnosis; (3)Only one of the four IHC staining procedures (OATP, GPC3, CS and HSP) was conducted. Such cases were excluded to ensure that the IHC diagnostic value was compared between the same lesions as much as possible to maintain homogeneity of analysis.

\section{Current used descriptive diagnosis}

Based on the evaluation of the location and needle path of the target lesions via preoperative grayscale ultrasonography, a 21-gauge fine needle (SONOPSY; Hakko, Tokyo, Japan) aspiration biopsy under ultrasonography or contrast enhanced ultrasound guidance was performed. At least two samples from each lesion and one sample from the adjacent liver tissue (negative control) were obtained. Formalin-fixed, paraffin-embedded tissue sections were consecutively cut and immunostained with antibodies directed against HE, VB, silver staining, CD34, and CK7 antigens.

We classified HCC into early, well, moderately, and poorly differentiated (diff.). The parameters evaluated in this study included cell atypia, structural atypia, reticular fiber patterns, neovascularization, stromal invasion, and ductular reaction (Table 1)[13]. The density of cancerous cells, nuclear size and morphology, nuclear/cytoplasmic ratio, and trabecular structure were observed via HE staining. Decrease of reticular fiber were evaluated using silver staining. By assistance of VB staining, intratumoral stromal invasion (tumor cell invasion into the intratumoral portal tracts [20]) for the diagnosis of early HCCs was determined [21]. We used the CK7 to stain the bile duct, which is a suggestive of change in bile metabolism in HCC [22]. CD34 was used to stain the endothelial cells to detect neovascularization [23]. Observation of CK7 staining was focus on the periportal bile duct of HCC lesions, which is reflection of dysfunction of bile-producing metabolic pathways[24, 25]. CD34 staining targeted at the cytoplasm/membrane of vascular endothelial cells, which reveal sinusoidal capillarization (Fig. 1-4). 
Table 1

Our currently used four-classification histological diagnostic criteria of $\mathrm{HCC}^{1}$

\begin{tabular}{|c|c|c|c|c|c|c|}
\hline \multirow{2}{*}{$\begin{array}{l}\text { Staining } \\
\text { method }\end{array}$} & \multirow{2}{*}{\multicolumn{2}{|c|}{ Estimated item }} & \multirow[t]{2}{*}{ Early HCC } & \multicolumn{3}{|l|}{ Advanced HCC } \\
\hline & & & & Well diff. & $\begin{array}{l}\text { Moderately } \\
\text { diff. }\end{array}$ & Poorly diff. \\
\hline \multirow[t]{6}{*}{ HE } & \multirow[t]{5}{*}{ Cell atypia } & Nucleus size & + & ++ & +++ & ++++ \\
\hline & & Nucleus morphology & Noncircular & Irregular & Deform & Bizarre \\
\hline & & Cellular density & + & ++ & +++ & ++++ \\
\hline & & $\mathrm{N} / \mathrm{C}$ & + & ++ & +++ & ++++ \\
\hline & & $\begin{array}{l}\text { Multinucleated giant } \\
\text { cells }\end{array}$ & - & - & + & ++ \\
\hline & $\begin{array}{l}\text { Structure } \\
\text { atypia }\end{array}$ & Trabecular structure & Thin & Thick+ & $\begin{array}{l}\text { Thick++/ } \\
\text { Solid+ }\end{array}$ & $\begin{array}{l}\text { Disappear/ } \\
\text { Solid++ }\end{array}$ \\
\hline Silver & Reticular fib & & Clear & Recognizable & Sparse & Disappear \\
\hline CD34 ${ }^{2}$ & Neovascula & & Negative/ focal & Diffuse & Diffuse & Diffuse \\
\hline$V^{3}{ }^{3}$ & Stromal inv & & Presence & Absence & Absence & Absence \\
\hline $\mathrm{CK} 7^{4}$ & Ductular rea & & $\begin{array}{l}\text { Decrease/ } \\
\text { Absence }\end{array}$ & Absence & Absence & Absence \\
\hline \multicolumn{7}{|c|}{$\begin{array}{l}1 \text { Abbreviations HCC: Hepatocellular Carcinoma; Diff.: Differentiated; HE:Hematoxylin and eosin staining; VB:Victoria Blue; CK: Cytokeratin; } \\
\text { N/C:Nuclear cytoplasmic ratio. }\end{array}$} \\
\hline \multicolumn{7}{|c|}{$\begin{array}{l}2 \text { Here, CD34 positive expression indicated stain in sinusoidal capillarization. "Negative" was defined as positive only for blood vessels and } \\
\text { bile ducts in portal tracts and/or rare sinusoidal spaces }(<10 \%) \text { near portal tracts. This pattern was observed in both early HCC and non-tumor } \\
\text { areas. "Focal" referred to a small section }(10-50 \%) \text { of the sinusoidal spaces showing immunoreactions with CD34. "Diffuse" meant that most } \\
\text { sinusoidal spaces }(>50 \%) \text { in the lesion areas were positive. }\end{array}$} \\
\hline \multicolumn{7}{|c|}{$\begin{array}{l}{ }^{3} \text { Here, positive staining of VB indicated stain portal tract in cancer lesion, thus presence of stromal invasion means presence of cancer cells } \\
\text { within portal tract. If portal tract invasion was recognized within the HCC lesion, grade of cancer cell atypia does not matter. }\end{array}$} \\
\hline
\end{tabular}

The final pathological diagnosis was made following a discussion between two pathological experts with $>20$ years' experience in liver pathology who were unaware of the results of the clinical laboratory examination and imaging diagnosis (M.O. and M.N.).

\section{Novel IHC markers-based diagnosis}

After HE, VB, CD34, CK7, and silver staining in the above step, IHC staining were performed using antibodies against OATP8, HSP70, GS, and GPC3 antigens, respectively. Negative controls consisted of reactions performed without primary antibodies, while positive controls were specimens containing antibodies in known cellular locations (e.g. nuclei, cytoplasm, membrane) and whose histomorphology and cytomorphology could be visualized via a "stain" [26].

According to a study by Sciarra, et al. [26], HSP70 (nuclei/cytoplasm) and GPC3 (cytoplasm/membrane) in cancer cells were scored as positive when unequivocally overexpressed, regardless of the staining intensity (Fig. 5). GPC3 (cytoplasm/membrane) was classified as "negative" (total percentage of positive cells $\leq 5 \%$ ), "focal positive" (5-50\% of cells stained), or "diffuse positive" (> 50\% of the cells stained) when making general comparisons among four histological grades (Fig. 5). However, GPC3 was divided into two groups when analyzing the efficacy of GPC3 in diagnosing a certain histological grade. GS expression in the cytoplasm of cancer cells and hepatocytes surrounding the terminal hepatic venules was determined. GS-positive areas were $<10 \%$ in cirrhotic livers, so staining in $\geq 10 \%$ of the liver was considered positive [27](Fig. 5).

Immunoreaction positivity of OATP was determined by staining the hepatocyte or tumor cell membrane, but not in terms of cytoplasmic reactivity, if present [28]. Staining was evaluated by two independent observers, with approximately five years of experience in liver pathology(M.T. and Y.I.). They were blinded to clinical and imaging information, especially histological grading diagnosis in terms of VB, silver staining, CD34 and CK7 immunostaining in previous steps. However, they were aware of HE staining outcomes, which they needed as a reference.

\section{Statistical analysis}


All the indicators we observed in this study (clinical characteristics, IHC indicators, and final pathological diagnoses) were analyzed. Continuous variable ages (with normal distribution) are presented as means \pm standard deviation (SD), and mean age was compared between histological grades using one-way analysis of variance (ANOVA). Continuous variable sizes (skewness distribution, homogeneity of variance) are described as medians (inter quartile range) and compared between different histology grades using the nonparametric Kruskal-Wallis test. The rest of the baseline data and imaging indicators were tested among the four histological groups using the chi-squared test and Fisher's exact test. Two-tailed Fisher's exact and chi-squared tests were used to compare the frequencies of all categorical variables for inter-group differences in histology grades. The area under the curve (AUC) of a receiver operating characteristic (ROC) curve was used to determine the diagnostic usefulness of indicators for each diff. HCC. The level of significance was set at $P<0.05$. The cut-off value of continuous data (lesion size) was obtained from the ROC curve analysis. The size value yielding the maximum sum of sensitivity and specificity is determined as the cutoff value. The above statistical analyses were performed using SPSS version 26.0 for Windows (SPSS Inc., Chicago, IL, USA). The sensitivity, specificity, and accuracy of each indicator were calculated manually.

\section{Results}

\section{Clinical characteristics}

Table 2 shows the clinical characteristics of four-grade histological classification of HCCs (patients and their corresponding lesions) collected in this study. There was no significant difference in general characteristics (age, gender, associated liver disease, Child-Pugh grade) among the patient groups. Lesion size was defined as the maximum diameter as measured by ultrasound (or contrast-enhanced ultrasound). The maximum diameter of eight lesions (>100 mm) significantly deviated from those of other lesions. Considering the possibility of large measurement error, these eight lesions were excluded as invalid values. Hence, the average tumor sizes in the four histological groups were $14 \mathrm{~mm}, 17 \mathrm{~mm}, 18 \mathrm{~mm}$, and $20 \mathrm{~mm}$, and they were significantly different from each other $(P<0.001)$.

Table 2

Clinical characteristics of HCC patients and lesions ${ }^{1}$

\begin{tabular}{|c|c|c|c|c|c|}
\hline & \multirow[t]{2}{*}{ Early HCC } & \multicolumn{3}{|c|}{ Advanced HCC } & \multirow[t]{2}{*}{$P$ value } \\
\hline & & Well diff. & Moderately diff. & Poorly diff. & \\
\hline No. of patients/lesions & $29 / 45$ & $24 / 31$ & $55 / 68$ & $49 / 56$ & / \\
\hline Age (mean $\pm S D$, years) & $70.3 \pm 13.5$ & $71.4 \pm 8.0$ & $70.1 \pm 8.9$ & $72.2 \pm 10.2$ & 0.493 \\
\hline Gender (Female/male) & $10 / 35$ & $6 / 25$ & $19 / 49$ & $11 / 45$ & 0.673 \\
\hline Etiology (HCV/HBV/NBNC) & $24 / 5 / 16$ & $19 / 4 / 8$ & $38 / 9 / 21$ & $30 / 11 / 15$ & 0.743 \\
\hline Child-Pugh grade (A/B) & $43 / 2$ & $24 / 7$ & $58 / 10$ & $47 / 9$ & 0.136 \\
\hline $\begin{array}{l}\text { Tumor size } \\
\text { [median, range, mm] }\end{array}$ & $14(12-16)$ & $17(15-20)$ & $18(15-24)$ & $20(12-27)$ & $<0.001$ \\
\hline
\end{tabular}

\section{Inter-group comparison of the IHC markers for four histological grades}

In general, when each diagnostic indicator was analyzed between any two histological grades, differences in GPC3, GS, HSP, and OATP8 expression were statistically significant between some, but not all histological grades (Table 3, $P$ value). Specifically, GPC3 clearly distinguished early from poorly diff. HCC from the other grades ( $P \leq 0.003)$. GS distinguished early HCC from the other grades $(P \leq 0.002)$. HSP70 clearly distinguished between poorly diff. and the other histological classifications $(P \leq 0.004)$. A difference was only observed between early and poorly diff. HCC in OATP8 $(P=0.019)$. 
Table 3

The relationship between IHC markers and histology classification of $\mathrm{HCC}^{1}$

\begin{tabular}{|c|c|c|c|}
\hline Indicators & Characteristics & Inter-grade comparison & $P$ value \\
\hline \multirow[t]{3}{*}{ GS } & \multirow[t]{3}{*}{ Negative or focal positive/diffuse positive } & Early $(11 / 33)$ and well $(0 / 30)$ & 0.002 \\
\hline & & Early and moderate $(3 / 65)$ & 0.001 \\
\hline & & Early and poorly $(1 / 52)$ & 0.001 \\
\hline OATP8 & $0 / 1 /(2 \text { and } 3)^{2}$ & Early(13/25/12) and poorly(24/1/11) & 0.019 \\
\hline \multirow[t]{5}{*}{ GPC3 } & \multirow[t]{5}{*}{ negative/focal positive/diffuse positive } & Early (40/4/1) and well (17/10/4) & 0.003 \\
\hline & & Early and moderate $(28 / 22 / 18)$ & 0.003 \\
\hline & & Early and poorly $(7 / 19 / 30)$ & $<0.001$ \\
\hline & & Poorly and moderate & 0.001 \\
\hline & & Poorly and well & $<0.001$ \\
\hline \multirow[t]{4}{*}{ HSP70 } & \multirow[t]{4}{*}{ negative/positive } & Early (37/7) and moderate (30/38) & $<0.001$ \\
\hline & & Poorly (17/36) and early & $<0.001$ \\
\hline & & Poorly and well (20/10) & 0.002 \\
\hline & & Poorly and moderate & 0.040 \\
\hline
\end{tabular}

\section{Analysis of the diagnostic efficacy of the IHC markers}

In general, some of the IHC indicators we studied performed well in the diagnosis of early HCC, followed by poorly diff. HCC (Table 4). Diagnostic efficacy for well and moderately diff. HCC was not very high (data not listed). In detail, optimal efficacy for early HCC detection was obtained when any two of the three IHC indicators GPC3, HSP70, and GS were negatively expressed. The sensitivity, specificity, accuracy, and AUC were $79.5 \%, 80.8 \%, 80.5 \%$, and 80.2 , respectively. For poorly diff. HCC, the combination of positive GPC3 and HSP70 expression yielded a relatively acceptable accuracy (74.4\%) and AUC (0.703). Although the negative expression of OATP8 was significant in the diagnosis of poorly diff. HCC (accuracy $=60.0 \%, A U C=0.566$ ), the combination of OATP8 in any form failed to improve the AUC and accuracy (accuracy $=67.3 \%, A U C=0.703$, data was not shown in tables). The highest efficacy for diagnosing well diff. HCC was the combination of positive HSP70 and GPC3 expression, while for moderately diff. HCC, the combination was of any two indicators of negative OATP expression and positive HSP and GPC3 expression. Unfortunately, their accuracy and AUC were all lower than 0.60 , inferior to those of early and poorly diff. HCC. 
Table 4

Diagnostic efficacy of IHC markers for different histological HCC ${ }^{1}$

\begin{tabular}{|c|c|c|c|c|c|c|c|c|c|}
\hline $\begin{array}{l}\text { Histological } \\
\text { grade }\end{array}$ & \multicolumn{2}{|c|}{ IHC indicators } & IHC patterns & $\begin{array}{l}\text { No. of } \\
\text { lesions }^{2}\end{array}$ & Sensitivity(\%) & Specificity(\%) & Accuracy(\%) & AUC (95\%Cl) & $\begin{array}{l}P \\
\text { value }\end{array}$ \\
\hline \multirow[t]{8}{*}{ Early } & \multicolumn{2}{|l|}{ GPC3 } & Negative & 200 & 88.9 & 66.5 & 71.5 & $\begin{array}{l}0.771(0.698- \\
0.844)\end{array}$ & $<.001$ \\
\hline & \multicolumn{2}{|l|}{ HSP70 } & Negative & 195 & 84.1 & 55.6 & 62.1 & $\begin{array}{l}0.699(0.616- \\
0.781)\end{array}$ & $<.001$ \\
\hline & \multicolumn{2}{|l|}{ GS } & Negative & 195 & 25.0 & 97.4 & 81.0 & $\begin{array}{l}0.612(0.509- \\
0.715)\end{array}$ & $<.001$ \\
\hline & \multicolumn{2}{|c|}{ GPC3 + HSP70 } & Both are negative & 195 & 77.3 & 81.5 & 80.5 & $\begin{array}{l}0.794(0.713- \\
0.874)\end{array}$ & $<.001$ \\
\hline & \multirow{3}{*}{$\begin{array}{l}\text { GPC3 } \\
+\mathrm{GS}+ \\
\mathrm{HSP} 70\end{array}$} & $\begin{array}{l}\text { Entire } \\
\text { lesions }\end{array}$ & All are negative & 194 & 23.3 & 98.7 & 82.0 & $\begin{array}{l}0.610(0.505- \\
0.714)\end{array}$ & $<.001$ \\
\hline & & $\begin{array}{l}\text { Size < } \\
18 \mathrm{~mm}\end{array}$ & $\begin{array}{l}\text { Any two are } \\
\text { negative }\end{array}$ & 106 & 76.3 & 80.9 & 79.2 & $\begin{array}{l}0.786(0.691- \\
0.881)\end{array}$ & $<.001$ \\
\hline & & $\begin{array}{l}\text { Size } \geq \\
18 \mathrm{~mm}\end{array}$ & $\begin{array}{l}\text { Any two are } \\
\text { negative }\end{array}$ & 81 & 100.0 & 81.3 & 82.7 & $\begin{array}{l}0.907(0.837- \\
0.976)\end{array}$ & $\begin{array}{l}< \\
0.001\end{array}$ \\
\hline & \multicolumn{2}{|c|}{$\begin{array}{l}\text { GPC3 + HSP70 + } \\
\text { GS }\end{array}$} & $\begin{array}{l}\text { Any two are } \\
\text { negative }^{3}\end{array}$ & 195 & 79.5 & 80.8 & 80.5 & $\begin{array}{l}0.802(0.724- \\
0.880)\end{array}$ & $\begin{array}{l}< \\
0.001\end{array}$ \\
\hline \multirow[t]{4}{*}{ Poorly } & \multicolumn{2}{|l|}{ GPC3 } & Positive & 200 & 90.6 & 59.2 & 67.5 & $\begin{array}{l}0.747(0.674- \\
0.819)\end{array}$ & $<.001$ \\
\hline & \multicolumn{2}{|l|}{ HSP70 } & Positive & 195 & 66.0 & 60.0 & 61.5 & $\begin{array}{l}0.630(0.541- \\
0.719)\end{array}$ & 0.001 \\
\hline & \multicolumn{2}{|l|}{ OATP8 } & Negative & 190 & 51.0 & 63.1 & 60.0 & $\begin{array}{l}0.566(0.472- \\
0.661)\end{array}$ & 0.083 \\
\hline & \multicolumn{2}{|c|}{ GPC3 + HSP70 } & Both are positive ${ }^{3}$ & 195 & 62.0 & 78.6 & 74.4 & $\begin{array}{l}0.703(0.615- \\
0.791)\end{array}$ & $<.001$ \\
\hline Well & \multicolumn{2}{|c|}{ GPC3 + HSP70 } & $\begin{array}{l}\text { None/focal } \\
\text { positive (GPC3) }+ \\
\text { negative(HSP70) }\end{array}$ & 198 & 56.7 & 59.5 & 59.1 & $\begin{array}{l}0.579(0.468- \\
0.691)\end{array}$ & 0.150 \\
\hline Moderately & \multicolumn{2}{|c|}{$\begin{array}{l}\text { OATP8 + GPC3 + } \\
\text { HSP70 }\end{array}$} & $\begin{array}{l}\text { Meet two } \\
\text { conditions }^{3,4}\end{array}$ & 196 & 55.2 & 58.1 & 57.1 & $\begin{array}{l}0.567(0.482- \\
0.652)\end{array}$ & 0.075 \\
\hline \multicolumn{10}{|c|}{$\begin{array}{l}{ }^{1} \text { HCC:Hepatocellular carcinoma; HSP70: heat shock protein 70; GPC3: Glypican 3; GS:glutamine synthetase; OATP:organic anion transport } \\
\text { peptide; AUC: area under the curve. Limited to the length of the manuscript, we do not list diagnostic results of all the IHC indicators and their } \\
\text { combinations, but only some with relative high accuracy and AUC. }\end{array}$} \\
\hline \multicolumn{10}{|c|}{${ }^{2}$ In some groups, the total number of cases was less than 200 , because some kind of IHC staining was not performed in a few patients. } \\
\hline \multicolumn{10}{|c|}{$\begin{array}{l}{ }^{3} \text { When using the combination of different IHC markers for diagnosis, we considered that this kind of combination was the most efficient } \\
\text { (because of the relative higher accuracy and AUC). }\end{array}$} \\
\hline
\end{tabular}

By analyzing the ROC curve of tumor size, we obtained the cutoff value of the tumor size as 17.5 mm in maximum diameter, which was used to divide lesions into two groups: the large size group (diameter $\geq 18 \mathrm{~mm}$ ) and the small size group (diameter $<18 \mathrm{~mm}$ ). Differences in the tumor sizes were significant only between early HCC and other histological groups $(P<0.001)$. Therefore, we took the lesion size as a subgroup for analyzing the diagnostic value of each indicator for early HCC. Consistently, the AUC and accuracy of the most valuable diagnostic indicators, (negative expression of any two of GPC3, HSP70, or GS), regardless of size, remained high after subgrouping. The AUC values for small and large size subgroups were 0.786 and 0.907 , respectively, and the accuracy values were $79.2 \%$ and $82.7 \%$, respectively (Table 4 ).

\section{Discussion}


Our research showed that the combination of some IHC indicators yielded good diagnostic accuracy for early and poorly diff. HCC. Early HCC was much easier to be detected than the other histological grades. Unfortunately, the diagnostic accuracies of each IHC indicator for well and moderately diff. HCC were not as good as those for early and poorly diff. HCC.

GPC3 is a member of the glypican family of heparinsulfate proteoglycans, which are cell proliferation inhibitors and apoptosis inducers [29]. GPC3 is negatively expressed in both normal liver tissue and benign hepatocellular nodules. Although GPC3 is not a specific hepatocellular marker, the reported sensitivity (91.7\%) and specificity (100\%) for small HCC detection are higher than those of alpha-fetoprotein (AFP). Thus, GPC3 is considered a reliable and early HCC biomarker [30]. Studies have reported that GPC3 overexpression increases with the progression of hepatocarcinogenesis [17, 31, 32].

HSP70 is a member of the HSP family, which is involved in the regulation of cell cycle progression and apoptosis. In early HCC, HSP70 synthesis may be stimulated by nutritional depletion and hypoxia resulting from insufficient blood supply [33]. Chuma et al. [33] reported the upregulation and significant overexpression of HSP 70 in early and advanced HCC, respectively.

Consistently with the promising findings from previous studies stated above, our study showed that GPC3 and HSP70 expression increased with the advancement of histological grade. Moreover, negative and positive GPC3 and HSP70 expression displayed good values for early and poorly diff. HCC, respectively. Nevertheless, some reports have suggested that it is difficult to diagnose poorly diff. HCC using IHC markers, because poorly diff. HCC may lose its immunoreactivity to some IHC markers [34]. In contrast, our study revealed that all the IHC markers we investigated were valuable for distinguishing poorly diff. HCC from other histological grades. Notably, GPC3 was positive in $87.5 \%$ (49 of 56) of poorly diff. HCC, similar to previous studies (85-89\%) [35]. Furthermore, it was exciting to find that the combination of GPC3 and HSP70 in our study yielded a relatively fair diagnostic value for poorly diff. HCC $(\mathrm{AUC}=0.703)$.

GS is an enzyme which supplies tumors (not specific to HCC) with energy by synthesizing glutamine. It has been speculated that as HCC progresses, the proliferation rate of tumor cells increases, and the expression of GS, which plays a key role in cell proliferation, increases accordingly [32]. Specifically, the degree (no, weak, moderate, or high) of GS expression has been reported as a sensitive marker of active $\beta$ catenin signaling in human HCC [36]. Nuclear expression of $\beta$-catenin might be more prone to occur in tumor cells of poorly diff. HCC, while $\beta$ catenin mutations are mainly found in non-HBV (hepatitis B virus), well diff., and chromosome-stable HCC [36]. Unfortunately, whether GS expression in relation to the histological de-differentiation of HCC is unclear, as published results are contradictory [31][32][37]. In our study, a positive GS outcome indicated that the GS expression level could distinguish early HCC from other histological grades. However, there was no significant difference between well diff. (negative/positive: 0/30), moderately diff. (0/68), and poorly diff. (1/52) HCC. Hence, there was no change in the distribution of negative and positive immunoreactivity between them. Unlike GPC3 and HSP70, the sensitivity of GS in the diagnosis of early HCC was extremely low (25\%). However, this does not mean that GS is useless for HCC grading differentiation. Surprisingly, GS specificity (97.4\%) was much higher than that of GPC3 (66.5\%) and HSP70 (55.6\%), suggesting that negative GS expression can almost rule out the possibility of advanced HCC. Taking advantage of the potential complementarity between GPC3 and HSP70, the combined application of these $\mathrm{IHC}$ indicators hint at the best efficacy for diagnosing early HCC (AUC $=0.802$, accuracy $=80.5 \%)$.

Many negative results were also noted for OATP8. As a member of the OATP family, which strongly mediates the transportation of a variety of endogenous and exogenous substrates through the cellular membrane, OATP8 (synonymous with OATP1B3, gene symbol SLC21A8) is specifically expressed at the basolateral hepatocyte membrane. OATPs are of considerable importance in HCC diagnosis and treatment, as they are closely related to the occurrence, recurrence, and prognosis of HCC [38]. However, only a few studies have explored the relationship between OATP expression and the histological grading of HCC. It is commonly acknowledged that, with increasing histological grading of HCC, bile formation decreases in the hepatocytes [11]. As OATP8 promotes hepatocyte recovery of bile acids from portal vein blood, OATP expression might theoretically be related to the histological classification of HCC. By semi-quantitatively evaluating the effect of OATP8 expression on the tumor cellular membrane relative to that on surrounding non-neoplastic hepatocytes, Kitao et al. reported that OATP8 expression significantly decreased from well to moderately to poorly diff. HCC [19], but the mechanism of this correlation remains unclear. However, the authors speculated that it may be attributable to the increased expression of hepatocyte nuclear factor (HNF)-3 $\beta$, an essential transcription factor for hepatocyte differentiation [19]. Tsuboyama et al. also found that as histological grade advanced from well diff. HCC to poorly diff. HCC, the dominant OATP distribution changed from grade 2 to grade 0 [28]. Unfortunately, the number of cases in this study was too small, and the authors did not make further statistical analyses or explain the changing trend in OATP. The method we used to detect OATP was exactly the same as that used by Kitao et al. [19], but a significant difference was only detected between early and poorly diff. HCC. It is unclear what makes our results different. Since there are few studies on the relationship between OATP and HCC histological grading, and more importantly, because whether used alone or in combination with other IHC markers, OATP contributed little to improve the diagnostic efficacy, we recommend OATP as an alternative supplementary rather than a preferred method for grading diagnosis.

In our study, we observed significant differences in lesion sizes between early HCC and other histological groups, suggesting that size may play a role in early HCC diagnosis. Some studies have reported that the histological grade of HCC progressed with the increase in tumor diameter [39] [40]. In view of this, we conducted a subgroup analysis of the most efficient diagnostic indicators for early HCC (combination of GPC3, HSP70, 
and GS) based on size. We found that the diagnostic efficiencies of both subgroups (big size group and small size group) were high, with an accuracy of $>79 \%$ and AUC of $>78 \%$. The statistical results were much closer to those not grouped by size $(A U C=0.802$, accuracy $=0.5 \%)$. These results suggest that size is a negligible factor in terms of the $\mathrm{IHC}$ indicator outcomes.

This study has a few limitations due to the specimen collection method. Compared with surgical resection, there might be a higher possibility of inadequate amount and heterogeneous staining of specimens obtained via fine needle aspiration biopsy [41], which may lead to errors in histopathological diagnosis. For example, once focal staining areas are undetectable in fractioned, tiny, and less representative tissue, they may be misdiagnosed as negative expression of immunohistochemical markers [31].

\section{Conclusion}

In conclusion, we comparatively analyzed IHC methods for distinguishing the histological stages of HCC. OATP, HSP70, GS, and GPC3, especially in certain combinations, produced considerate differential effects between different histological grades and further demonstrated good diagnostic ability in early and poorly diff. HCCs. Therefore, we recommended IHC markers-based diagnostics method as a promising option instead of currently used descriptive method mainly based on cell and structural atypia.

\section{Abbreviations}

HCC: Hepatocellular carcinoma; CK: Cytokeratin; HE: Haematoxylin and eosin; VB:Victoria blue; IHC: Immunohistochemistry; HSP:Heat shock protein; GPC3:Glypican 3; GS: Glutamine synthetase; OATP: Organic anion transport peptide; Diff.: Differentiated; AUC: Area under the curve.

\section{Declarations}

\section{Ethics approval and consent to participate}

This study was conducted with the approval of institutional review board of Medical Center of Yokohama City University (approval number: B180200054).

\section{Consent for publication}

Not applicable.

\section{Availability of data and materials}

The datasets used and/or analyzed during the current study are available

from the corresponding author on reasonable request.

\section{Competing interests}

The authors declare that they have no competing interests.

\section{Funding}

Not applicable.

\section{Authors' contributions}

M.C. and H.N. contributed to study design and data collection. A.N. and S.M. analyzed the data. M.N. designed the study and reviewed the slides. M.T., M.O. (Masako Otani) and Y.I. provided tissue microarray slides, reviewed the slides, and helped with immunohistochemistry. F.W. W.L. and L.R. wrote the original draft. M.O.(Masahiro Okada) and S.M. reviewed and edited the manuscript. K.N. supervised the research and finalized the manuscript. All authors read and approved the final manuscript.

\section{Acknowledgements}

Not applicable.

\section{References}

1. Farinati F, Sergio A, Baldan A, et al. Early and very early hepatocellular carcinoma: When and how much do staging and choice of treatment really matter? A multi-center study. BMC Cancer, 2009. 9: 33. 
2. Inoue K, Takayama T, Higaki T, et al. Clinical significance of early hepatocellular carcinoma. Liver Transpl, 2004. 10(2 Suppl 1): S16-19.

3. Yamamoto M, Takasaki K, Otsubo T, et al. Favorable surgical outcomes in patients with early hepatocellular carcinoma. Ann Surg, 2004. 239(3): 395-99.

4. Tamura S, Kato T, Berho M, et al. Impact of histological grade of hepatocellular carcinoma on the outcome of liver transplantation. Arch Surg, 2001. 136(1): 25-30, 31.

5. Takayama T, Makuuchi M, Hirohashi S, et al. Early hepatocellular carcinoma as an entity with a high rate of surgical cure. Hepatology, 1998. 28(5): 1241-46.

6. Sasaki K, Matsuda M, Ohkura Y, et al. In hepatocellular carcinomas, any proportion of poorly differentiated components is associated with poor prognosis after hepatectomy. World J Surg, 2014. 38(5): 1147-53.

7. Sciarra A, Di Tommaso L, Nakano M, et al. Morphophenotypic changes in human multistep hepatocarcinogenesis with translational implications. J Hepatol, 2016. 64(1): 87-93.

8. Kondo F. Histological features of early hepatocellular carcinomas and their developmental process: For daily practical clinical application : Hepatocellular carcinoma. Hepatol Int, 2009. 3(1): 283-93.

9. International Working Party. Terminology of nodular hepatocellular lesions. Hepatology, 1995. 22(3): 983-93.

10. International-Consensus-Group-for-Hepatocellular-Neoplasia. Pathologic diagnosis of early hepatocellular carcinoma: A report of the international consensus group for hepatocellular neoplasia. Hepatology, 2009. 49(2): 658-64.

11. Edmondson H, Steiner P. Primary carcinoma of the liver: A study of 100 cases among 48,900 necropsies. Cancer, $1954.7(3)$ : $462-503$.

12. Hamilton S R, Aaltonen L A, World Health Organization, International Agency for Research on Cancer. Pathology and genetics of tumours of the digestive system. 2000: Lyon Oxford: IARC Press; Oxford University Press. 314.

13. Wang F, Numata K, Nakano M, et al. Diagnostic value of imaging methods in the histological four grading of hepatocellular carcinoma. Diagnostics (Basel), 2020. 10(5).

14. Wang F, Numata K, Nakano M, et al. Diagnostic value of imaging methods in the histological four grading of hepatocellular carcinoma. Diagnostics (Basel), 2020. 10(5): 321.

15. Roskams T, Kojiro M. Pathology of early hepatocellular carcinoma: Conventional and molecular diagnosis. Semin Liver Dis, 2010. 30(1): 1725.

16. Yao S, Zhang J, Chen $\mathrm{H}$, et al. Diagnostic value of immunohistochemical staining of GP73, GPC3, DCP, CD34, CD31, and reticulin staining in hepatocellular carcinoma. J Histochem Cytochem, 2013. 61(9): 639-48.

17. Shirakawa $\mathrm{H}$, Kuronuma T, Nishimura $\mathrm{Y}$, et al. Glypican-3 is a useful diagnostic marker for a component of hepatocellular carcinoma in human liver cancer. Int J Oncol, 2009. 34(3): 649-56.

18. Nguyen T B, Roncalli M, Di Tommaso L, et al. Combined use of heat-shock protein 70 and glutamine synthetase is useful in the distinction of typical hepatocellular adenoma from atypical hepatocellular neoplasms and well-differentiated hepatocellular carcinoma. Mod Pathol, 2016. 29(3): 283-92.

19. Kitao A, Matsui O, Yoneda N, et al. The uptake transporter OATP8 expression decreases during multistep hepatocarcinogenesis: Correlation with gadoxetic acid enhanced MR imaging. Eur Radiol, 2011. 21(10): 2056-66.

20. Nakano M, Saito A, Yamamoto M, et al. Stromal and blood vessel wall invasion in well-differentiated hepatocellular carcinoma. Liver, 1997. 17(1): 41-46.

21. Kobayashi S, Kim S R, Imoto S, et al. Histopathological diagnosis of early HCC through biopsy: Efficacy of Victoria blue and cytokeratin 7 staining. Dig Dis, 2012. 30(6): 574-79.

22. Park Y N, Kojiro M, Di Tommaso L, et al. Ductular reaction is helpful in defining early stromal invasion, small hepatocellular carcinomas, and dysplastic nodules. Cancer, 2007. 109(5): 915-23.

23. Maeda T, Adachi E, Kajiyama K, et al. CD34 expression in endothelial cells of small hepatocellular carcinoma: Its correlation with tumour progression and angiographic findings. J Gastroenterol Hepatol, 1995. 10(6): 650-54.

24. Tummala K S, Brandt M, Teijeiro A, et al. Hepatocellular Carcinomas Originate Predominantly from Hepatocytes and Benign Lesions from Hepatic Progenitor Cells. Cell Rep, 2017. 19(3): 584-600.

25. Barakauskiene A, Speiciene D, Liakina V, et al. Expression of cytokeratin 7 as a histological marker of cholestasis and stages of primary biliary cirrhosis. Medicina (Kaunas), 2011. 47(1): 31-38.

26. Hewitt S M, Baskin D G, Frevert C W, et al. Controls for immunohistochemistry: The Histochemical Society's standards of practice for validation of immunohistochemical assays. J Histochem Cytochem, 2014. 62(10): 693-97.

27. Sakamoto M. Early HCC: Diagnosis and molecular markers. J Gastroenterol, 2009. 44 Suppl 19: 108-11.

28. Tsuboyama T, Onishi H, Kim T, et al. Hepatocellular carcinoma: Hepatocyte-selective enhancement at gadoxetic acid-enhanced MR imagingcorrelation with expression of sinusoidal and canalicular transporters and bile accumulation. Radiology, 2010. 255(3): 824-33. 
29. Filmus J. Glypicans in growth control and cancer. Glycobiology, 2001. 11(3): 19R-23R.

30. Zhang Q, Xiao Q, Lin Z, et al. Development of a competitive radioimmunoassay for glypican-3 and the clinical application in diagnosis of hepatocellular carcinoma. Clin Biochem, 2010. 43(12): 1003-08.

31. Di Tommaso L, Franchi G, Park Y N, et al. Diagnostic value of HSP70, glypican 3, and glutamine synthetase in hepatocellular nodules in cirrhosis. Hepatology, 2007. 45(3): 725-34.

32. Wasfy R E, Shams E A. Roles of combined glypican-3 and glutamine synthetase in differential diagnosis of hepatocellular lesions. Asian Pac J Cancer Prev, 2015. 16(11): 4769-75.

33. Chuma M, Sakamoto M, Yamazaki K, et al. Expression profiling in multistage hepatocarcinogenesis: Identification of HSP70 as a molecular marker of early hepatocellular carcinoma. Hepatology, 2003. 37(1): 198-207.

34. Kaseb A O, Hassan M, Lacin S, et al. Evaluating clinical and prognostic implications of Glypican-3 in hepatocellular carcinoma. Oncotarget, 2016. 7(43): 69916-26.

35. Nguyen T, Phillips D, Jain D, et al. Comparison of 5 immunohistochemical markers of hepatocellular differentiation for the diagnosis of hepatocellular carcinoma. Arch Pathol Lab Med, 2015. 139(8): 1028-34.

36. Zucman-Rossi J, Benhamouche S, Godard C, et al. Differential effects of inactivated Axin1 and activated beta-catenin mutations in human hepatocellular carcinomas. Oncogene, 2007. 26(5): 774-80.

37. Shin E, Ryu H S, Kim S H, et al. The clinicopathological significance of heat shock protein 70 and glutamine synthetase expression in hepatocellular carcinoma. J Hepatobiliary Pancreat Sci, 2011. 18(4): 544-50.

38. Li T T, An J X, Xu J Y, et al. Overview of organic anion transporters and organic anion transporter polypeptides and their roles in the liver. World J Clin Cases, 2019. 7(23): 3915-33.

39. Kenmochi K, Sugihara S, Kojiro M. Relationship of histologic grade of hepatocellular carcinoma (HCC) to tumor size, and demonstration of tumor cells of multiple different grades in single small HCC. Liver, 1987. 7(1): 18-26.

40. Huang K, Dong Z, Cai H, et al. Imaging biomarkers for well and moderate hepatocellular carcinoma: Preoperative magnetic resonance image and histopathological correlation. BMC Cancer, 2019. 19(1): 364.

41. Di Tommaso L, Destro A, Seok J Y, et al. The application of markers (HSP70 GPC3 and GS) in liver biopsies is useful for detection of hepatocellular carcinoma. J Hepatol, 2009. 50(4): 746-54.

\section{Figures}



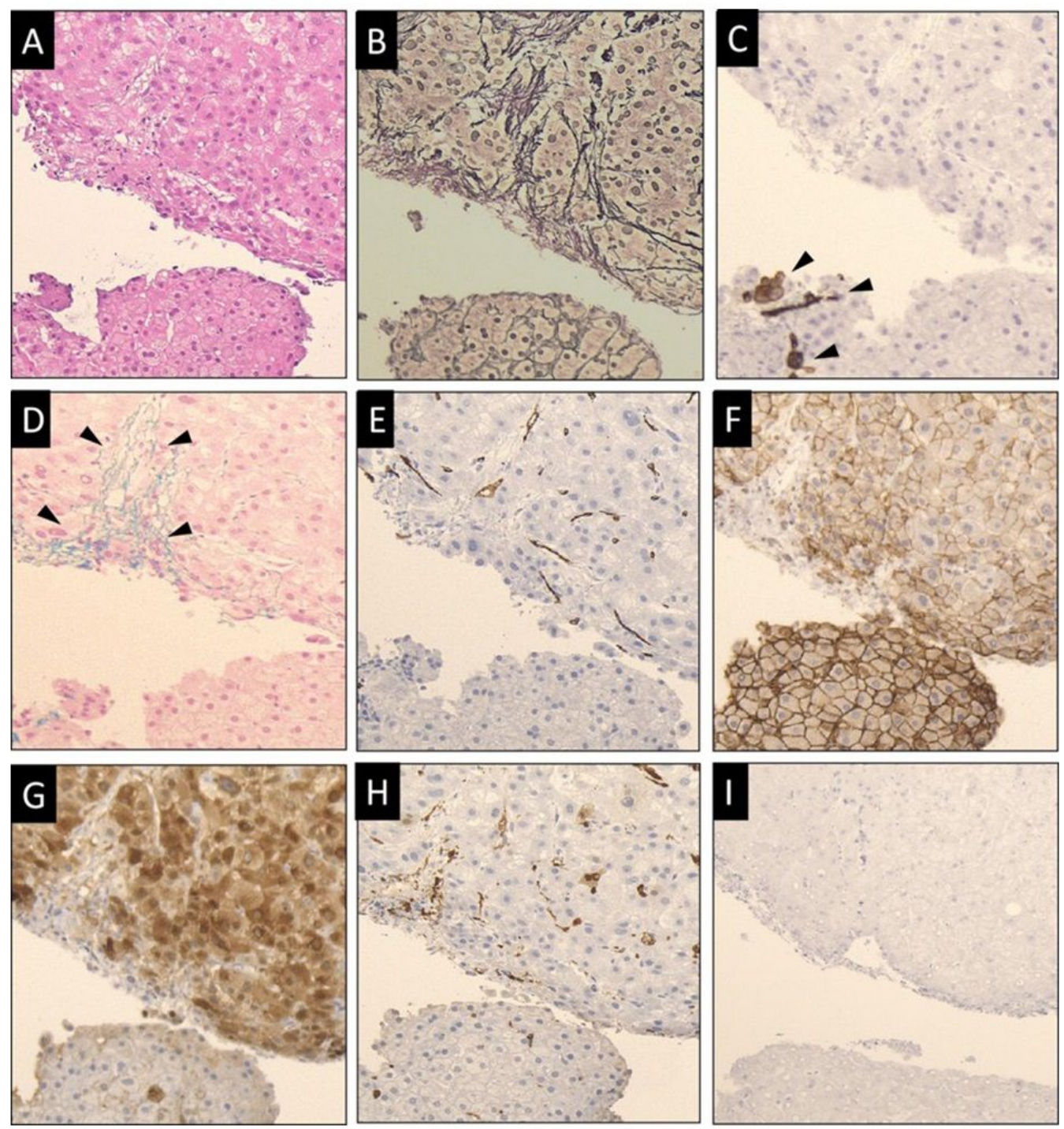

Figure 1

Histological and IHC diagnosis of early HCC. A) In hematoxylin-eosin (HE) staining, compared with the non-tumor area, cancer area shows slight cell atypia such as an increased nuclear cytoplasmic ratio, noncircular nucleus and mild hyper-cellularity. The trabeculae are arranged clearly with normal trabecular structure. B) Ag staining of reticular fibers formation is slightly unclear in tumor areas when compared with non-tumor areas. C) In Cytokeratin 7 staining, the stroma within cancer area shows no ductular reaction while the non-tumor area has ductular reaction (arrowheads) in the peripheral area of portal tract. D) Victoria blue staining shows the stroma within tumor area clearly (arrowheads). E) Focal positive expression of CD34 is present in sinusoidal capillarization in cancer area while no expression is shown in non-tumor areas. F) In the cell membrane, cancer cells in tumor area exhibit slightly positive OATP expression while hepatocytes in non-tumor area strongly positive expression. G) Diffuse positive expression of GS is shown in cytoplasmic of cancer cells while almost none in non-tumor area. H) In both cancer cells and non-tumor hepatocyte, no HSP70 are expressed in cytoplasmic, thus negative expression of HSP70 is determined. I) In both cancer and non-tumor area, the expression of GPC3 are negative.The upper part of the figures are tumor areas while the lower part are non-tumor areas. 

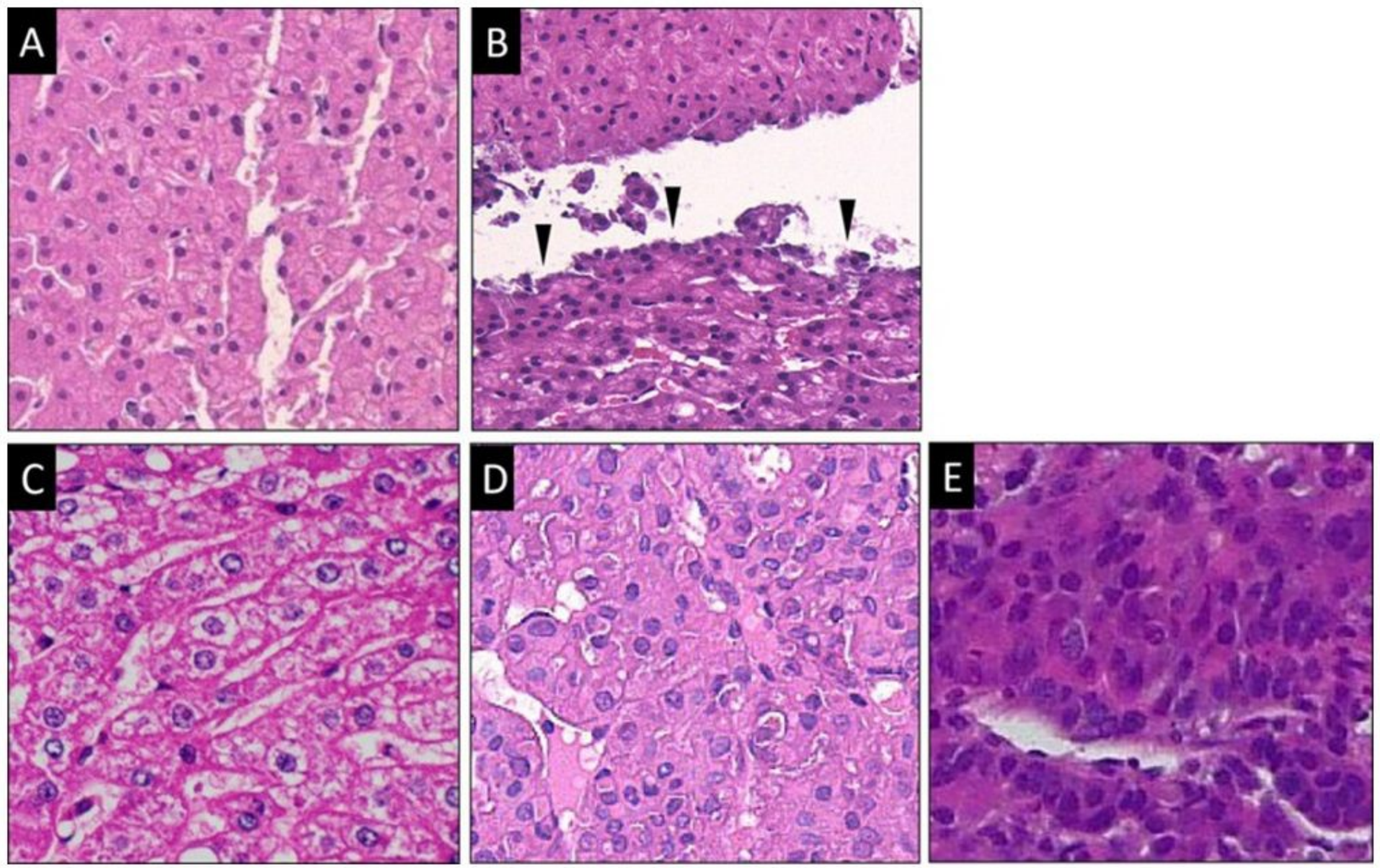

Figure 2

Hematoxylin and eosin (HE) staining of liver biopsy specimen. A) In non-tumor area, normal hepatocytes show the normal nucleus shape and nuclear cytoplasmic ratio. Thin trabeculae is clearly seen. B) In early HCC, the cancer area (arrowhead) have slightly cell atypia compared with the non-tumor area (the upper half). The cancer area has an appearance of increased nuclear cytoplasmic ratio, mild density of tumor cells and slight large, noncircular nucleus. C) In well diff. HCC, increased density of tumor cells, increased nuclear cytoplasmic ratio and large nuclei with irregular shape can be seen. Trabecular structure distorted into as thick as two cell layers. D) Moderately diff. HCC shows obvious cell atypia and abnormal trabecular structure. In details, hyper-cellularity and larger, irregular shapes of nucleus can be seen. Nuclear cytoplasmic ratio is increased more significantly. A few of multinucleated giant cancer cells can be seen. Trabecular structure still exists but thick and distorted in display. E) In poorly diff. HCC, the phenomenon of hyper-cellularity is even more serious. Large, bizarre shaped nucleus are commonly seen. There are many multinucleated giant cancer cells. Nuclear cytoplasmic ratio is increased more significantly. The tumor cells commonly solid in architecture and have no trabecular structure. 

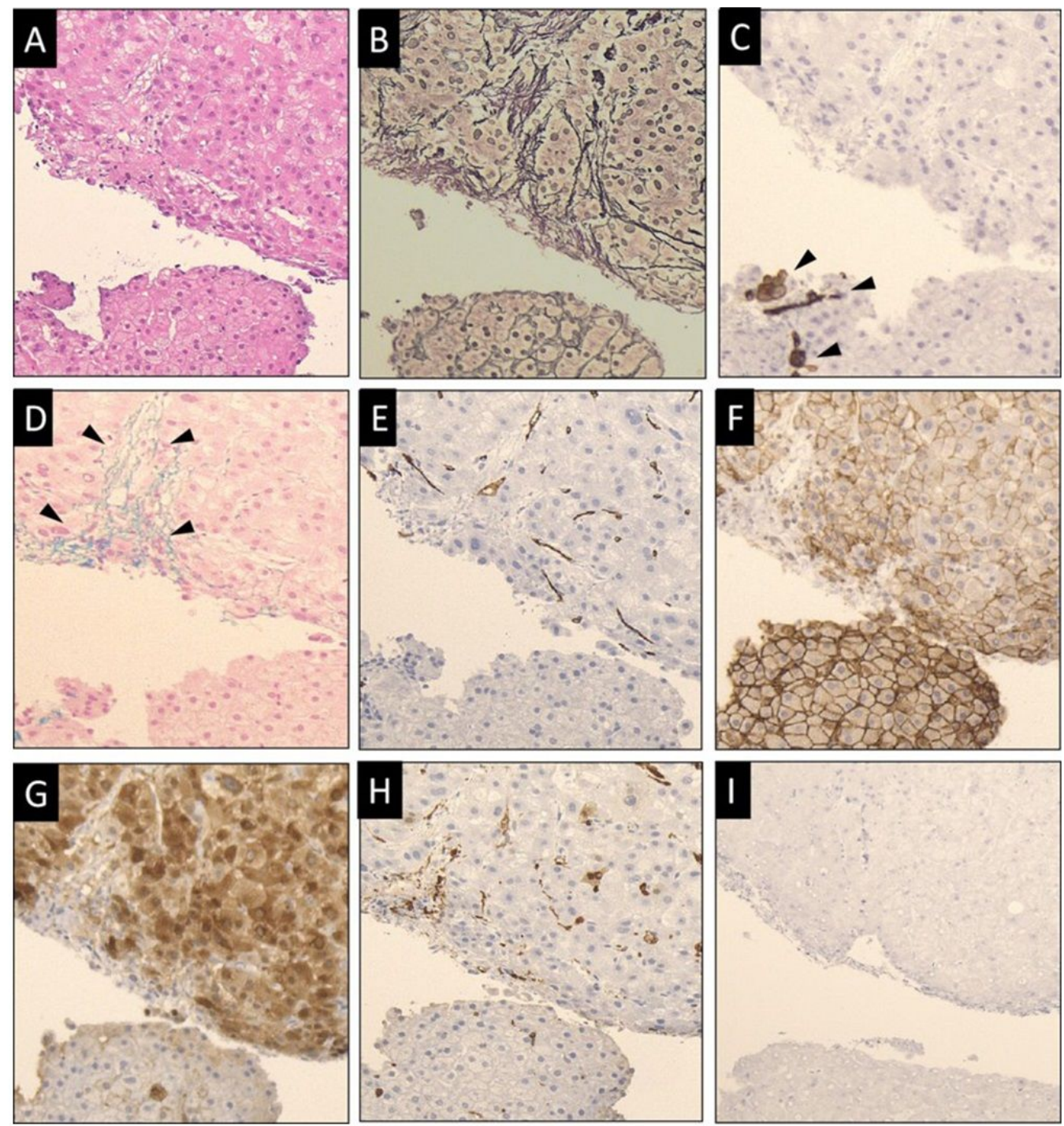

Figure 3

Silver staining of liver biopsy specimen. A) In non-tumor area, reticular fibers distribute clear and evenly. They show normotrabecular pattern. B) In early HCC, reticular fibers distribute clear and evenly. They show two cell thick layer pattern. C) In well diff. HCC, reticular fibers are recognizable clearly. D) In moderately diff. HCC, reticular fibers are sparsely distributed and not very clear. E) In poorly diff. HCC, reticular fibers are totally disappeared. 

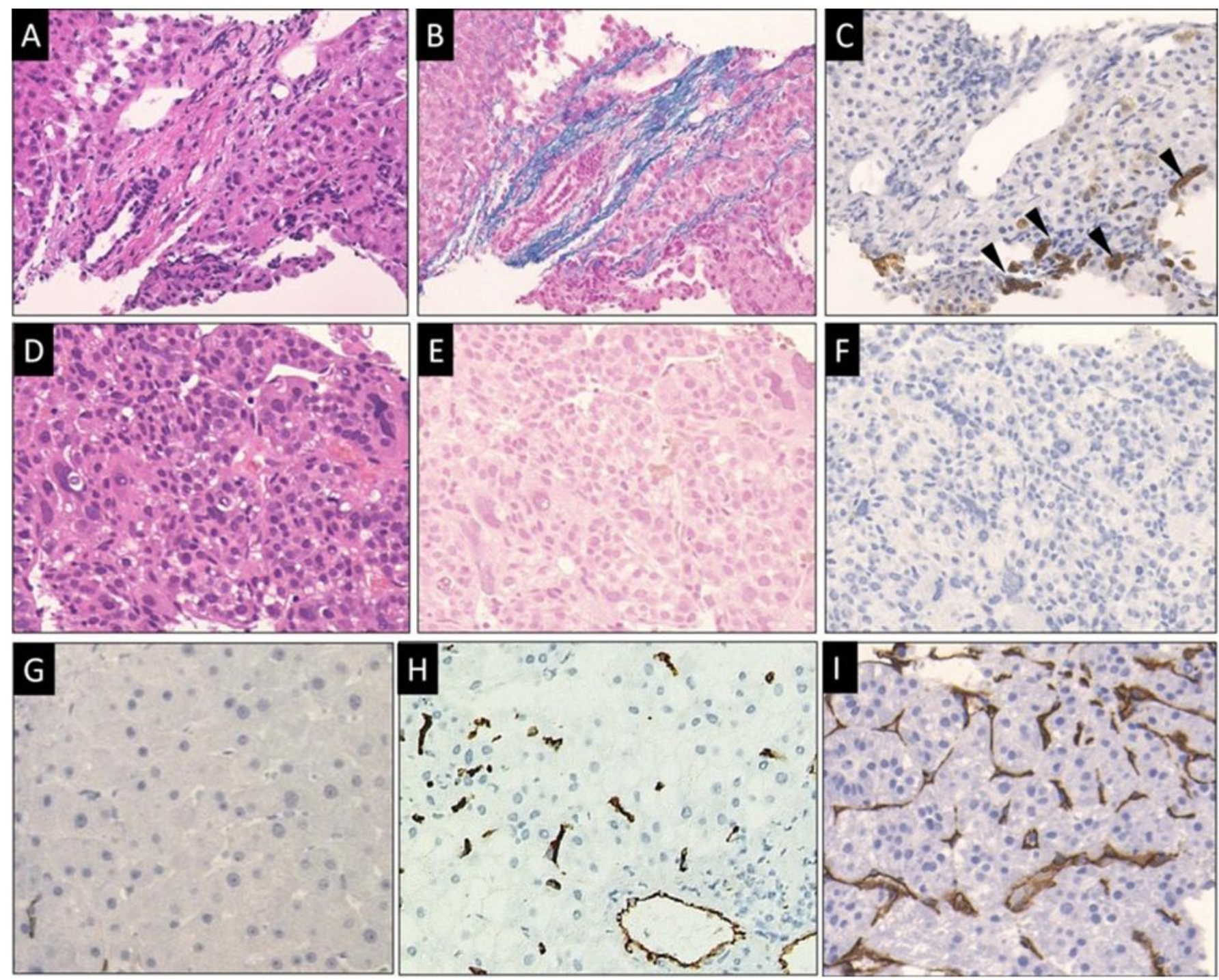

Figure 4

Victoria blue, Cytokeratin7 and CD34 staining of liver biopsy specimen. A) The HE staining of an early HCC case is taken as a reference for Victoria blue and Cytokeratin 7. B) Compatible with a diagnosis of early HCC, Victoria blue staining shows elastic fibers in the stroma in blue. Deformed cancer cells are seen within the stroma. This finding means positive stromal invasion. C) In early HCC, using Cytokeratin 7 staining, slight ductular reaction is observed in the right side of the periportal area adjacent to the stroma (portal tract) (black arrowheads). In contrast, ductular reaction is absent in the left side. D) The HE staining of a moderately diff. HCC case is taken as a reference for Victoria blue and Cytokeratin 7. E) In moderately diff. HCC, Victoria blue is negatively expressed in tumor area. F) In Cytokeratin 7 staining of moderately diff. HCC, the stroma within cancer area shows no ductular reaction. G) The Negative expression of CD34 in non-tumor area. H) In early HCC, a few of peripheral bile ducts and portal tract are positive immunoreactivity, indicating focal CD34 expression. I) In moderately diff. HCC, strong diffuse expression of CD34 suggests increased neovascularization, resulting from sinusoidal capillarization and formation of sinusoid vascular endothelium in HCC. 

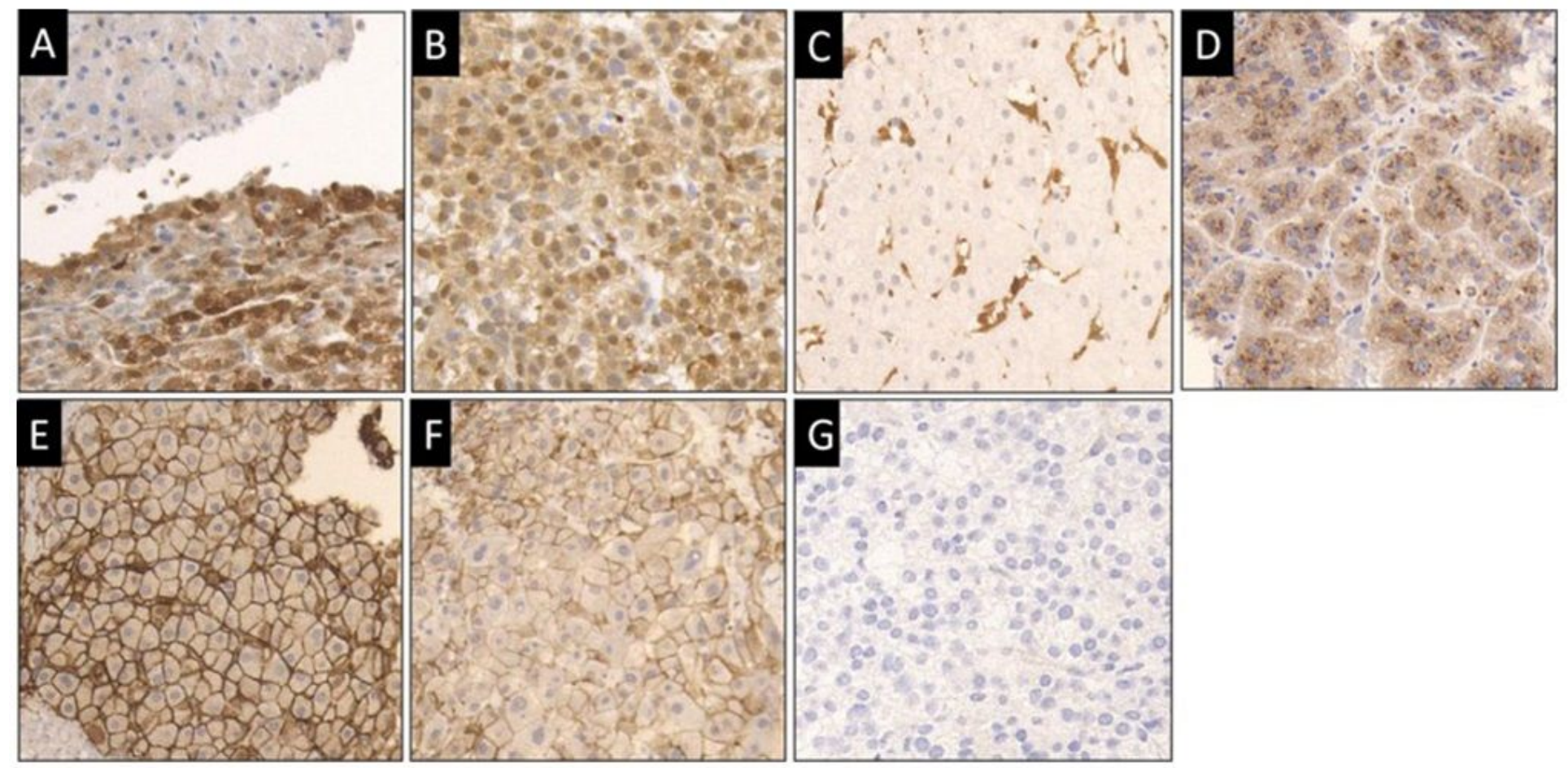

Figure 5

Staining grading of immunohistochemical markers. A) Negative expression in non-tumor area (upper) and positive expression in tumor area (lower) of Glutamine synthetase (GS) in hepatocyte cytoplasm. B) Diffuse cytoplasmic and nucleo immunoreactivity of HSP 70. C) In non-tumor area, only Kupffer cell is stained of HSP 70 while there is no cytoplasmic and nucleo expression, so it is defined as HSP70 negatively expression. D) This figure shows glypican 3 (GPC3) is diffusely positive expression in cytoplasm and membranous of HCC cells in a case with poorly diff. HCC. E) This figure shows strong positive stain of organic anion transport peptide (OATP) 8 in almost all part of membrane of hepatocytes in nontumor area. F) In a case with a moderately diff. HCC, positive stain of OATP 8 is decreased. G) This figure shows no expression of OATP8 in membrane in a case with poorly diff. HCC. 